\title{
Is a detection of 'Free-Floating' Chemical Properties Possible?
}

\author{
Bernd Lindemann* \\ FR Physiologie, Universität des Saarlandes, Germany
}

Received: January 17, 2018; Published: January 25, 2018

*Corresponding author: Bernd Lindemann, FR Physiologie, Universität des Saarlandes, 66421 Homburg, Germany, Tel: 49684162349;

Email: mail@Bernd-Lindemann.de

\section{Abstract}

The claim that in olfaction objectless properties are detected treats abstract properties as if they were concrete objects, it is reification. Primary events of olfactory perception, leading to a population message indicating odour quality, involve a detection of molecular objects owning properties, not of objectless properties.

Keywords: Olfaction; Objectless Properties; Reification; Sensory Data; Neuronal Messages; Percepts; Philosophy

\section{Introduction}

Philosophers interested in the sense of smell sometimes wondered whether the odorants are detected as physical objects or as 'free-floating' properties of such objects [1,2]. Here I argue, following Bunge and Mahner [3] that 'free-floating' properties, i.e. properties not owned by a physical object, cannot exist. Starting with this ontological insight, a coarse roadmap of olfactory processing may be generated [4,5], which sheds light on the role of object-less properties by taking account of results of sensory physiology, aided by a philosophical appreciation of the concepts used. I World of concrete interacting objects. In olfaction it is the proximal olfactory object, consisting of volatile molecules dissolved in a thin layer of mucus, which interacts with the molecular receptors of olfactory sensory neurons and triggers transduction. Note that such interaction is not achieved by free-floating properties unattached to the object. Explanation: A physical object, e.g. a real object of classical mechanics, is a property-bearer constituted and characterised by its properties [6-8]. The object is concrete, defined in space and time, able to interact with other objects, thereby changing its set of properties. However, its properties are not concrete, a property has neither neither space nor time, cannot interact on its own.

Ontological argument against "objectless properties": Properties, being attributes of objects, do not exist independently like objects. Attributes cannot occur "object less" in reality [3]. Logical argument against "objectless properties": Properties can be expressed as predicates. The predicate in brackets (red-is-aproperty-of)-x becomes part of a meaningful statement only when $\mathrm{x}$ is substituted with the name of a red object: For the predicate is about the object, it does not make sense without the object to which it relates e.g. [9]. To assign to attributes features of objects would be a category mistake. This particular fallacy is known as reification [10-12], because an abstract (the attribute) is treated as if it were concrete (could exist independently, like an object). II. World of neuronal messages and processing. Generally, one type of odour molecule (object) bears several chemical properties which (as a part of the object) trigger several types of olfactory receptors mounted on many neurons. There by the object is detected through some of its features.

Olfaction characteristically does not carry spatial information about the stimulus. Such information is prominent in other senses, where receptor cells and receptors are arranged in spatial maps. In the olfactory mucosa the arrangement of receptor neurons is contingent, does not support the processing of spatial properties [2]. Following detection, subsequent events of transduction generate neuronal messages which convey sensory data. As always, the data consist of a rapidly variable part and a related stationary part. The rapidly variable part is spike-encoded and travels along the afferent nerve fibres to the olfactory bulb. It conveys quantity (intensity) and answers the question "How much?" [13]. However, the fibres are 'labelled lines' e.g. [14]. which convey additional information with the label, which is the stationary part. The stationary part of the data is given by receptor specificity and neuronal connectivity (literature in) [15]. It specifies which type of receptor was activated and implies that the receptor activated has 
interacted with a physical object of sufficient binding affinity. Thus the primary message transported by a single sensory nerve fibre (point 6) does not only carry intensity information, it also conveys information on connectivity and the receptor specificity. The full message may amount to a statement (in a simple case) like: "An object with property xy of intensity $\mathrm{z}$ is detected"[1].

Note that detected is an object because only an object can trigger a receptor interactively and that [2] the object is not specified, except for the one observation that it has property $x y$. The sensory input provided by many fibres, on occasion rapidly changing, results in a constantly updated activity spectrum or across-fibre-pattern [e.g. 16]. This 'fingerprint' may be realised by a plot of the variable vs. the stationary part of the data. At any instant of time the first-neuron-pattern reveals numerous detected properties of the odour molecules, amounting to odour quality. The pattern is based on the output of millions of neurons. After convergent processing in the olfactory bulb the output pattern at this stage describes the present "quality" of the smell, based on a few hundred projection neurons only. At both stages the quality message carries information about an object interacting through its properties with multiple receptors. The object is not missing; it is the topic of the sensory messages. Sensory quality relates an object owning a set of properties to a mental experience. Its two aspects are [1]. By the detected properties of a physical object, quality identifies the object. It can be taken as data about the object. Data, however, being about an object, cannot be "free-floating" or "objectless" [2]. Quality is associated with a peculiar experience, a quale (see "percept", below).

The properties detected are not all the properties of the molecular object. Such incompleteness, however, is a necessary feature of any sensory perception. III. World of mental phenomena, experience of virtual objects. Following neuronal processing, the sensory messages give rise to the mental experience of a percept. This, when restricted to one sense, is properly classified as "monomodal". The "smell of a rose" is an example. It stands for the detected molecular binding properties of the odorant molecules. The combination of such properties defines the quality, which is made conscious as a mental experience, a smell. Percepts are understood as mental reconstructions, recreations or models of real objects [17]. Yet a smell does not inform the smeller about molecular binding properties, of course. Rather, the set of properties detected is tagged with a peculiar "raw feel" or "quale". This is a unique conscious experience [18] which invokes emotions, draws on memory and is specific for the sub-modality in question. There is a striking difference in qualia tagging perceptions like "sweet", "salty", "blue", "smell of Chanel no 5", "smell of H2S" etc., as revealed by introspection.

The content of messages from several senses, aided by memory, is mentally experienced. As a multimodal percept, i.e. "a rose" rather than "the smell of a rose". The multimodal percept stands for the physical object providing the stimuli for smell, vision, touch etc. The multimodal experience is cast in a set of qualia encoding the modalities and sub-modalities involved in the symbolic, image-rich or anyway non-verbal and emotion-raising idiom of the mental. Physical objects are ontologically real, of course. Also real are neuronal mechanisms, including those processing sensory data and those generating mental phenomena (3rd person perspective). However, the mental phenomena generated, which supervene over neuronal mechanisms, are not independently real (1st person perspective). Those mental phenomena which, like percepts, represent real objects, may be classified as "virtual objects". If the virtual object (or representation) is successfully modelling the real object, then ontological rules (like in point 2) may also apply to virtual objects. In other words, mentally experienced properties and qualities are virtual properties and qualities owned by virtual objects modelling real objects. They, too, are not "objectless" or "free-floating".

\section{Conclusion}

Does it possible, then, that "objectless qualities" rather than molecular objects own qualities are detected? [1] Taking qualities as attributes of a physical object, the question must be denied because according to the ontological and predicatelogical points raised above, objectless attributes cannot exist on their own and do not make sense on their own [2]. Taking receptor events as critical for detection, only objects, not objectless properties can interact with receptors [3]. Taking quality as neuronal data about an object (point 10) it appears that data are always related to an object, they are about a thing and cannot be "objectless".

\section{References}

1. Batty C (2010) A Representational account of olfactory experience. Canadian Journal of Philosophy 40: 511-538.

2. Keller A (2016) Philosophy of Olfactory Perception. palgrave, NewYork, USA, p.85.

3. Bunge MA, Mahner M (2004) Über die Natur der Dinge. Hirzel, Stuttgart, Germany, p. 22.

4. Lindemann B (2017) Objects, Properties, Percepts: a Philosopher's Sniff. Chemical Senses 42(9): 789-7791.

5. Lindemann B (2018) Olfaction, Philosophy, and the Missing Object. Chemical Senses 43: in press.

6. Bundle theory

7. Physical body

8. Physical property

9. Predicate logic

10. Whitehead AN (1925) Science and the Modern World (The Lowell Lectures). Reprint 1967, Free Press, Macmillan Publishing Co Inc, New York, USA, p. 51.

11. List of fallacies

\section{Reifikation}

13. Foerster H von (1985) Sicht und Einsicht. Versuche zu einer operativen Erkenntnistheorie, Carl-Auer, Heidelberg, Germany, pp.1999.

14. Erickson RP (1963) In Sensoryneural patternsand gustation.Zotterman $Y$, (Edr). Olfaction and Taste, Oxford Pergamon Press, pp. 205-213.

15. Lindemann B (2014) Mechanisms in World and Mind. Imprint academic, Exeter, UK, p. 64. 
16. Pfaffmann C (1959) The afferent code for sensory quality. Am Psychol 14(5): 226-232.

\section{(C) (i) This work is licensed under Creative}

Submission Link: http://biomedres.us/submit-manuscript.php
17. Reconstruction

18. Qualia 